\title{
OBITUARY
}

\section{Raymond Davis Jr (1914-2006)}

Father of solar neutrino detection.

For 30 years, Ray Davis could have been mistaken as a miner. Clad in hard hat, headlamp and battery belt, he would join 50 other 'first shifters' for a pre-dawn ride a mile into the Earth. A labyrinth of hot, dimly lit tunnels greeted the riders, but Davis knew his way, having retraced it some 700 times. Turning to the right, he headed towards the tunnel with the bright lights. At the end, beneath those lights, was a 380,000-litre tank. This was where he did his mining. While his companions blasted tonnes of granite in search of gold, Davis sifted through 600 tonnes of cleaning fluid for a dozen special atoms much more interesting to him than gold.

His was the more difficult task. No mining engineer would accept the odds - find 10 atoms in a sea of $10^{31}$ - nor would workers a third his age long want to keep pace. He spent 15 hours a day underground each time he came to Homestake and, consequently, often wouldn't see the Sun. However, if you asked him about it, he would laugh, flash a broad smile and say, "I'm watching the Sun all the time!" Ray Davis, who died on 31 May, had indeed been watching the Sun in a new and interesting way since 1967. By doing so, he helped discover something unexpected in particle physics.

It was in the late 1930s that Hans Bethe provided the first rigorous explanation of why the Sun shines. Nuclear reactions in the core fuse hydrogen into heavier elements, with light being the most obvious (but not the only) by-product. Another particle, the neutrino, was also produced and, unlike light, travelled virtually unhindered from the solar interior. Wolfgang Pauli postulated this particle in 1930 to preserve several sacred laws of physics, but in the same breath dismissed it as probably undetectable given its tiny cross-section and resulting elusive nature. His worry was unfounded. In the 1950s, Fred Reines and Clyde Cowan saw signs of this poltergeist at the Savannah River reactor, an intense source of (anti)neutrinos.

Davis also began his neutrino work at reactors. He had earned a PhD at Yale, served in the army during the war, and worked briefly as a radiochemist at Monsanto, before joining the newly created Brookhaven National Laboratory in 1948 - his home for the next 37 years. When he asked the chemistry department chairman for his assignment, he was told to go to the library and find something interesting to work on. He did. A review paper on neutrinos caught his eye, as did a technical brief by Bruno Pontecorvo. Pontecorvo proposed a way

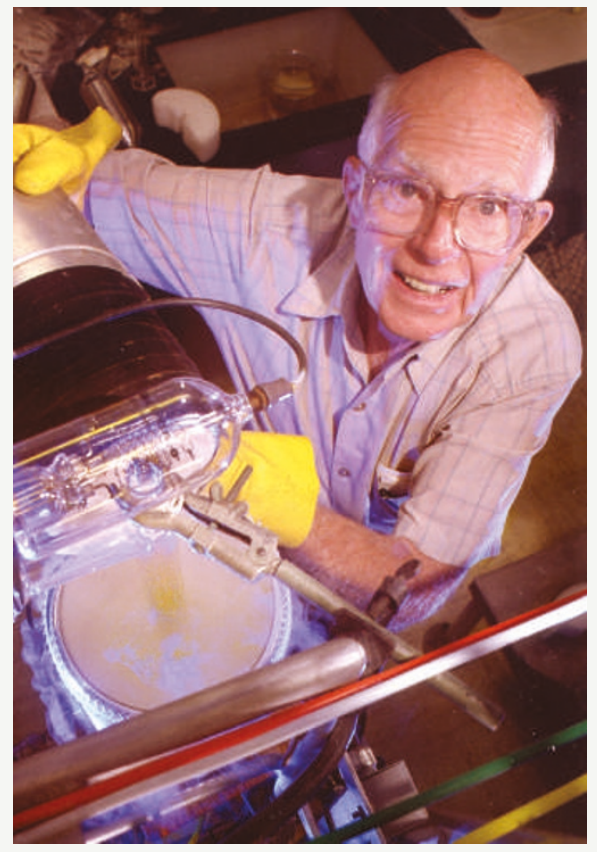

of detecting neutrinos using a chlorinecontaining liquid. The idea was that a neutrino would be captured on a particular chlorine isotope $\left({ }^{37} \mathrm{Cl}\right)$, changing it into a radioactive argon atom $\left({ }^{37} \mathrm{Ar}\right)$, which could be removed from the liquid and counted. Davis fleshed out the scheme, developing the extraction, gas handling and counting hardware needed to make it work. He constructed a 3,800-litre detector and looked for argon production at both the Brookhaven and Savannah River reactors. He found none - not because of any experimental failings, but because of his emerging finding that neutrinos and antineutrinos were different - at least with respect to capture on ${ }^{37} \mathrm{Cl}$. In 1960, he began thinking about a grander challenge - detecting neutrinos from the Sun.

In this quest, Davis found an able guide in John Bahcall. Bahcall, a theorist, began calculating neutrino emissions from the Sun and their capture rate on chlorine. Davis was proposing a scaled-up 380,000-litre detector filled with $\mathrm{C}_{2} \mathrm{Cl}_{4}$ (a dry-cleaning fluid). Bahcall predicted that, of the more than $10^{22}$ neutrinos transiting this detector each week, about 10 would be captured, creating 10 argon atoms a week. Though small, it was a rate that Davis thought he could measure. Not one to make an idle boast, he had been working to improve the technologies for this large-scale detector and to understand its background. From earlier measurements, he knew that his detector would have to be deep underground to shield it from cosmic rays. After a brief search for a site, the detector was built at the Homestake Gold Mine in South Dakota from 1965 to 1967.

A problem arose almost immediately when data started arriving. Davis's measurements were about a third of Bahcall's prediction. In spite of more refined calculations and more data, the discrepancy remained. Dubbed the 'solar neutrino problem', it persisted for 20 years. Davis and his experiment remained in the crosshairs the entire time. He was challenged repeatedly - about efficiencies, chemical traps, pump changes, and the like - but he faced all questioners with his trademark candour, graciousness and wit. He brought technical precision and thoroughness to his answers, recounting the many careful, quantitative tests he had performed to address each concern. He had deep humility, but also quiet confidence in his experimental skills - which were formidable.

Beginning in the late 1980s, other experiments came online. They confirmed the problem. In the end, a versatile detector in Canada resolved it, showing that neither Bahcall's calculation nor Davis's mining was mistaken. Instead, neutrinos from the Sun were changing 'flavour' en route to Earth. This unexpected result meant that neutrinos, formerly thought to be massless, necessarily had some - a finding that required revision of the standard model of particle physics and gave these mysterious messengers a mass on a par with all visible matter in the Universe.

In 2002, Davis won the Nobel Prize in Physics. His wife of 54 years, Anna, and 21 other Davises accompanied him to Stockholm. Then 88 and battling Alzheimer's, Ray received an additional honour. Nobel protocol ordinarily requires each recipient to walk to the centre of the stage to receive their prize. However, on this occasion the King of Sweden came in person to a laureate. The gold that Davis had never sought, never mined for, found him that day.

Those of us who worked alongside Ray Davis, at Brookhaven and later at the University of Pennsylvania, saw up-close a great experimentalist - heroically persistent, patiently tenacious and meticulously precise. But we saw an even greater human being one whose kindness, warmth and modesty were even more remarkable.

\section{James R. Distel}

James R. Distel is at Los Alamos National Laboratory, Los Alamos, New Mexico 87545, USA e-mail: jdistel@lanl.gov 